\title{
Política de dividendos y conflicto en la empresa familiar: el protocolo
}

\section{Dividend policy and conflict in family firms: the prolocol}

\author{
Virginia Llorente Muñoz \\ * Departamento de Contabilidad y Gestión. Universidad de Málaga, 29071 (Spain)
}

D A T OS A RTÍCULO

\section{Historial:}

Recibido 19-02-2012

Aceptado 24-05-2012

Palabras clave:

Conflicto

Empresa Familiar

Protocolo

Códigos JEL:

M41

\section{A R T ICLE INFO}

Article history:

Received 19 February 2012

Accepted 24 May 2012

Keywords:

Conflict

Family Business

Protocol

JEL codes:

M41

\section{RES U M EN}

El presente trabajo trata de analizar la política de dividendos y el conflicto en la empresa familiar. Se muestra la utilidad del desarrollo del protocolo familiar tras una revisión de la literatura. Se sugieren líneas futuras de investigación y se destaca la importancia del protocolo para la empresa familiar discutiendo las implicaciones para los distintos subsistemas de la empresa familiar.
A B S T R A C T

One of the issues that family business research strives to know is the influence of the dividend policy in the family business conflict. In this paper we analyze the family business protocol and conflict in the business. This papers aims to give guidance on the development of the family business protocol. After a review of the state of affairs, important research gaps are identified, and future research priorities are suggested. Implications for the family and business systems are discussed.

\footnotetext{
* Autor de contacto.

Correoselectrónicos:vlm@uma.es
} 


\section{Introducción}

La empresa familiar es una organización de compleja, ya que en ella deben convivir los sistemas empresa y familia. Desde 1961 y especialmente en los últimos años, la investigación en la empresa familiar ha alcanzado gran un desarrollo abriéndose camino como campo de estudio en la Dirección de Empresas (Chrisman et al., 2008), aunque existe aún una falta de consenso en la definición del concepto de empresa familiar (Litz, 1995; Miller et al., 2007).

La presencia de la empresa familiar en las economías nacionales y en el mercado global ha despertado, en las últimas décadas el desarrollo de la disciplina (Basco, 2010).

Desde 1989 la disciplina entró en el proceso de desarrollo de sus fonteras de investigación (Casillas and Acedo, 2007). Wortman (1994) concreta el comienzo de la disciplina a finales de la década de los setenta. Según Handler (1989), Neubauer y Lank (1998) se inicia sobre 1985 y especialmente 1990 como las fechas que marcan el inicio de la consolidación del estudio de la empresa familiar como disciplina (Benavides, Quintana, Guzmán, 2011).

Basco (2010) expone que según el Instituto de Empresa Familiar, en España, existen aproximadamente un millón y medio de empresas familiares en España que representan el $75 \%$ del empleo privado. El sistema empresa y el sistema familia no sólo coexisten en la empresa familiar sino que son interdependientes por lo que los conflictos pueden ser más frecuentes en concreto los de índole económica.

El trabajo se estructura de la siguiente forma. La primera sección es de carácter introductorio. En la segunda sección se analiza el reparto de dividendos y la política de dividendos. La siguiente sección se dedica a la análisis del conflicto, estudiándose los diferentes tipos de objetivos contradictorios de los distintos subsistemas. Finalmente se analiza el protocolo como herramienta para reducir el conflicto y por último se presentan las conclusiones principales del trabajo, las futuras líneas de investigación y las limitaciones del mismo.

\section{Reparto de dividendos}

Guzmán (2004) expone que la política de dividendos de las empresas ha sido un tema comúnmente tratado por diferentes investigaciones, llegando a calificarse de verdadero puzzle o rompecabezas (Black,

\section{Tabla 1}

Trabajos destacados sobre política de dividendos.

\begin{tabular}{ll}
\hline $\begin{array}{l}\text { Miller y Modigliani } \\
(1961)\end{array}$ & La política de dividendos es irrelevante sobre el valor de las acciones. \\
\hline Gordon (1959) & La política de dividendos tiene un efecto sobre el valor de las acciones \\
\hline $\begin{array}{l}\text { Poterba yummers (1985) } \\
\text { Allen et al. (2000) }\end{array}$ & Análisis bajo la perspectiva de las teorías fiscales. \\
\hline $\begin{array}{l}\text { Bhattacharya (1979) } \\
\text { Lang y Litzenberger } \\
(1989)\end{array}$ & Teoría informativa. \\
\hline $\begin{array}{l}\text { Easterbrook (1984) } \\
\text { Jensen (1986) } \\
\text { Rozeff (1982) }\end{array}$ & Enfoque de la teoría de la agencia. \\
\hline $\begin{array}{l}\text { Gugler (2003) } \\
\text { Gugler y Es Yortoglu } \\
(2003)\end{array}$ & $\begin{array}{l}\text { Se observa un menor reparto de dividendos en empresas bajo control familiar } \\
\text { en comparación con las no familiares. }\end{array}$ \\
\hline Jian-Ping y Yong (2005) & $\begin{array}{l}\text { Observan que cuando los accionista controladores poseen altos niveles de } \\
\text { control en la empresa, suele reducirse la cuantía de los dividendos que se }\end{array}$ \\
reparten.
\end{tabular}


1976).

Diversos trabajos analizan las repercusiones de la estrategia del reparto de dividendos en los precios de las acciones, mientras que otros estudian los factores influyentes en la adopción de la decisión de repartir beneficios. La tabla 1 resume algunos importantes trabajos.

Lozano, De Miguel y Pindado (1998) exponen que los problemas de agencia entre los agentes empresariales y los conflictos derivados en ocasiones se minimizan si existe por parte de los directivos participación en el capital, tal y como justifican diversos estudios (Demsetz y Lehn, 1985; Fama y Jensen 1983, Jensen y Meckling, 1976), teniendo en cuenta que pueden sentirse más proclives a el esfuerzo, a buscar de proyectos de inversión rentables o la incorporación de nuevas tecnologías (Jensen y Meckling, 1976).

Además, las empresas familiares suelen tener cierta resistencia al cambio (Levinson, 1987) y suelen restringir la participación de las nuevas generaciones en los procesos de toma de decisiones (Eddleston and Kellermanns, 2007; Stavrou, 1999).

Los conflictos de intereses entre gerentes y accionistas pueden aumentar si no existen objetivos comunes.

La participación de los directivos en la propiedad de la empresa suele reducir la tendencia a emplear el cash flow libre en proyectos de inversión no rentables y suele minimizar la sobreinversión (Jensen, Solberg y Zorn, 1992; Noronha, Shome y Morgan, 1996).

Teniendo en cuenta que un objetivo financiero importante de la empresa es aumentar el valor de mercado de las acciones, es importante que las empresas formulen su propia política de dividendos, incluido el caso extremo de aquellas que excepcionalmente deciden no pagar ningún dividendo.

El protocolo puede constituir una útil herramienta para concretar la política de dividendos y en general planificar y clarificar la dinámica de la empresa familiar.

\section{Conflicto en la Empresa Familiar}

El hecho de que la propiedad se encuentre en manos de la familiar puede originar conflictos de poder especialmente importantes ya que se mezclan los conflictos de la empresa con los de la familia, no obstante, en lo referente a la creación de valor, la empresa familiar muestra un mejor comportamiento que las no familiares, lo que pudiera explicarse por la existencia de menores conflictos de agencia (Santana y Cabrera, 2001).

Las empresas familiares implican un alto nivel de interacción entre trabajo y familia, con la consecuencia del traslado de los problemas familiares al trabajo y viceversa (Rosenblatt, 1991; Danes et al., 1999).

Familia y negocio están tan relacionados en las empresas familiares que el potencial del conflicto es mayor que en otro tipo de empresas (Lee and Rogoff, 1996). Los conflictos en las empresas familiares son una combinación de conflictos relacionados con el negocio y familiares (Harvey and Evans, 1994). El conflicto se menciona con frecuencia como una potencial desventaja de las empresas familiares (Levinson, 1971).

Sin embargo, si revisamos la literatura sobre el conflicto en la empresa familiar podemos comprobar que el conflicto no es inherentemente negativo, puede traer consigo consecuencias positivas. Múltiples trabajos señalan los beneficios del conflicto para las empresas familiares (Sharma et al., 1997; Kellermanns and Eddleston, 2004).

Por lo tanto, la comprensión y la gestión del conflicto en negocios familiares pueden ser factores críticos a corto plazo para la productividad del negocio y a largo plazo para su viabilidad y supervivencia (Sadler, 1990; Danes et al., 1999).

El conflicto relacionado con el trabajo puede proporcionar información importante para afrontar cambios de del entorno y tomas de decisiones (Kellermanns and Eddleston, 2004), lo que es un aspecto muy importante dado que el conocimiento necesario para tomar decisiones complejas excede a menudo los límites de las habilidades individuales (Walsh and Fahey,

Llorente Muñoz, V. (2012). Política de dividendos y conflicto en la empresa familiar: el protocolo. Revista de Empresa Familiar, 2(1), 5563 
1986). El conflicto puede traer consecuencias negativas, pero el conflicto constructivo relacionado con el trabajo puede ser importante para el funcionamiento de este tipo de empresas.

El conflicto relacionado con el trabajo puede mejorar la calidad de la toma de decisiones, los planes estratégicos, el crecimiento de la organización y su funcionamiento financiero (Schweiger et al., 1989).

El conflicto puede causar un desequilibrio en la empresa y si no se consigue resolver puede provocar la desaparición del negocio familiar (Harvey and Evans, 1994). De hecho, un conflicto de relaciones interpersonales con emociones negativas como el resentimiento y la animosidad daña el funcionamiento de la empresa familiar (Eddleston and Kellermanns, 2007).

La permeabilidad de los sistemas empresa y familia afecta las motivaciones sobre las que se justifica la existencia de la empresa familiar y crea un conjunto de objetivos relacionados (Basco, 2010).

En el caso de la empresa familiar resulta habitual que el control ejercido por los propietarios familiares no se limite únicamente a su participación en la propiedad, al jugar éstos generalmente un papel activo en la dirección (La Porta et al., 1999). En las empresas familiares podemos encontrar objetivos económicos y también otros de índole no económica (Chrisman, Chua y Sharma, 2005; Astrachan y Jaskiewicz, 2008).

Anderson et al. (2003) argumentan que los accionistas bajo lazos familiares suelen tener comportamiento diferentes a otros accionistas de otras empresas no familiares en al menos dos aspectos: la preocupación por la supervivencia de la empresa a largo plazo y la importancia de la reputación familiar.

En una empresa familiar pueden existir distintos grupos de interés y cada grupo puede tener objetivos que lógicamente difieran del resto. El clásico esquema de Tagiuri y Davis (1996) y el análisis de los objetivos en la empresa familiar (Tagiuri y Davis, 1992) nos proporciona una visualización de las tres dimensiones que componen una empresa familiar: propiedad, familia y empresa. En la empresa familiar es muy frecuente el

\section{Figura 1}

Objetivos y sistemas en la empresa familiar.

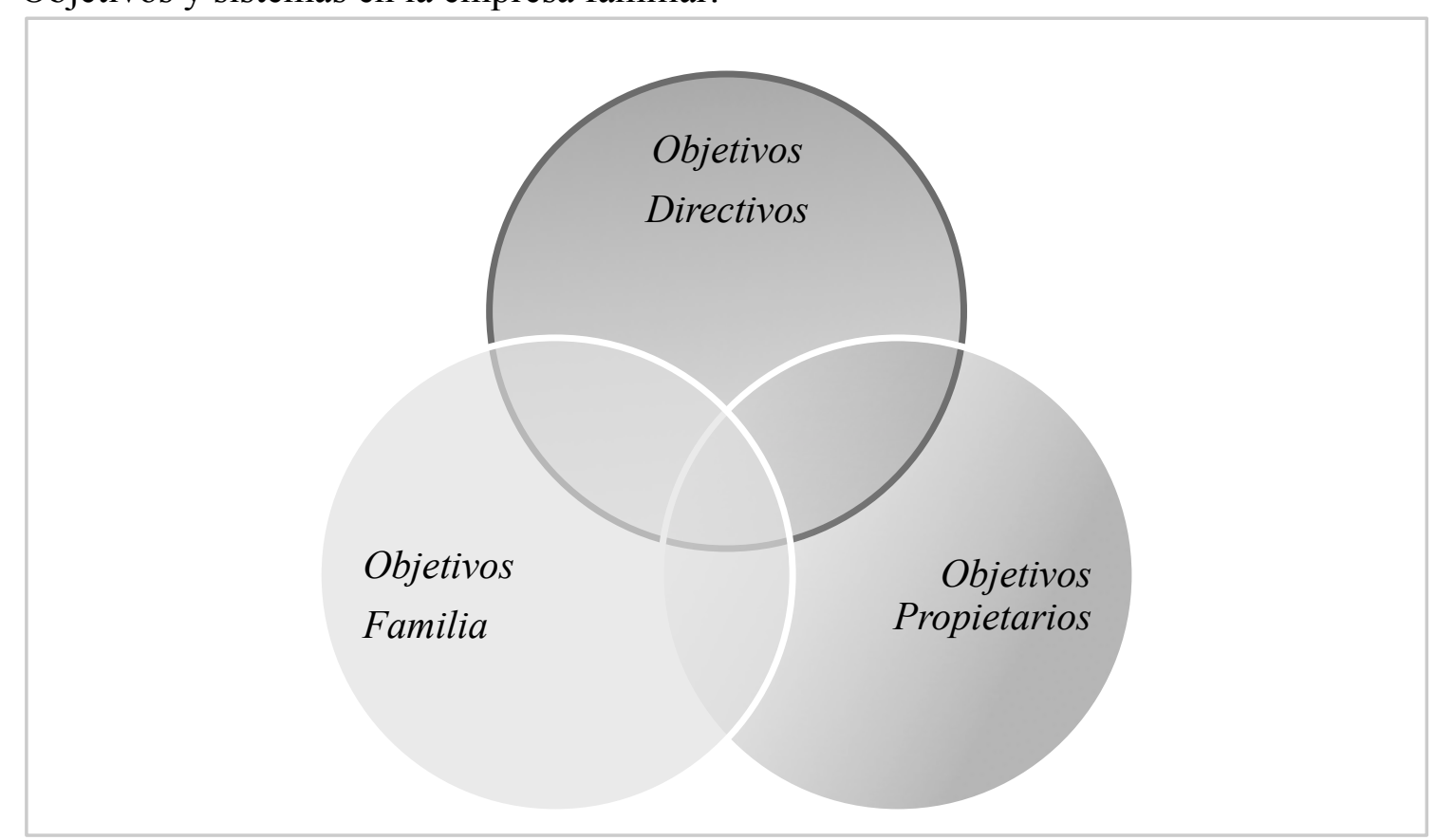

Fuente: elaboración a partir de Tagiuri y Davis (1996) 
solapamiento de roles, de forma que el gerente en diversas ocasiones es también propietario y miembro familiar. Teniendo en cuenta este hecho los grupos se conformarán según el citado esquema representado en la figura 1 en los siguientes:

-Objetivos de los miembros de la empresa familiar que son únicamente propietarios, aquellos miembros de la empresa que no son familia de los propietarios familiares de la empresa ni trabajan en ella. Al no poseer relaciones afectivas de tipo familiar, suelen tener objetivos relacionados con la rentabilidad, tal y como Sabater (2010) expone en el caso que la participación sea mediante acciones es frecuente que deseen dividendos, más que reservas.

-Objetivos de los miembros de la familia, aquellos familiares que no tienen propiedad, ni gestionan la empresa. Sabater (2010) expone que sus objetivos estarán relacionados con la obtención de dividendos frente reservas por preferir la rentabilidad en el presente.

-Objetivos de los gerentes (directivos no familiares) que no tienen participación en la propiedad, ni son miembros de la familia. Suelen tener objetivos de crecimiento (Tagiuri y Davis, 1992) y de continuidad de la empresa familiar.

-Objetivos de los miembros de la empresa familiar que son propietarios y familiares, pero no trabajan en la empresa: suelen estar relacionados con la obtención de una rentabilidad presente y por tanto tendentes al reparto de dividendos.

-Los objetivos característicos de miembros de la empresa familiar que son propietarios, trabajan en la empresa, pero no son familiares. Suelen tener objetivos de crecimiento (Tagiuri y Davis, 1992) y también de continuidad de la empresa familiar.

-Miembros de la familia que trabajan en la empresa pero no tienen propiedad en la misma tendrán sus objetivos particulares. Suelen tener igualmente objetivos de crecimiento $y$ de continuidad.

-Miembros que reúnen los tres requisitos: son propietarios, son miembros de la familia y trabajan en la empresa tendrán diversos objetivos interrelacionados.

La mejor solución entre dividendos $\mathrm{y}$ reservas dependerá evidentemente de las circunstancias empresariales y familiares, lo importante es el acuerdo entre los distintos grupos que coexisten en la empresa familiar. Es recomendable que los miembros abran el debate y lleguen a un acuerdo por consenso que pueda plasmarse en el protocolo familiar.

Debemos tener en cuenta que al producirse el reparto de dividendos entre los socios, se produce un elevado coste fiscal. Nos encontramos ante la doble imposición de los dividendos (Gallo y Vilaseca, 1996), los cuales van a tributar en el Impuesto sobre Sociedades de la entidad que los reparte, la cual habrá de realizar la correspondiente retención, pero que además van a tributar en el Impuesto sobre la Renta de los perceptores. La deducción por doble imposición intersocietaria viene a paliar los efectos de la doble imposición, pero no la elimina en su totalidad.

Una eficaz herramienta para la minimización de conflictos es el protocolo como analizaremos a continuación.

\section{El protocolo}

El protocolo familiar es un instrumento que regula las relaciones entre los miembros de la familia y la empresa (Sanchez-Crespo, 2003).

Diversos aspectos relativos a la publicidad de los protocolos familiares están en el caso de España regulados. El protocolo puede ser publicado en de forma voluntaria en el Registro Mercantil y en los sitios Web de las sociedades (Real Decreto 171/2007 de 9 de febrero).

Dicho Real Decreto se dictó en ejecución del mandato de la disposición final segunda, apartado 3, de la Ley 7/2003, de 1 de abril, de la sociedad limitada nueva empresa que dispone: "reglamentariamente se establecerán las condiciones, forma y requisitos para la publicidad de los protocolos familiares, así como, en su caso, el acceso al registro mercantil 
de las escrituras públicas que contengan cláusulas susceptibles de inscripción".

El protocolo familiar es un documento escrito constituido por un conjunto de pactos o códigos de conducta que suscriben los miembros del grupo familiar para garantizar la permanencia de la empresa en el que se concretan diversos aspectos como los valores, la relación entre familiares y sus políticas con respecto a la empresa.

-Gestión de los recursos humanos: procedimientos de evaluación de capacidades, procedimientos de selección, sistemas de remuneración, evaluación del desempeño, etc.

-Política de dividendos

-Transmisión acciones

-Órganos de gobierno: tanto de la familia como de la empresa.

\section{-Planificación estratégica}

-Gestión de los conflictos en la organización.

-Cómo se va a realizar el proceso de sucesión sucesión y traspaso generacional.

-Gestión de los directivos no familiares.

-Ética y resposabilidad social

-Misión y valores de la empresa

-Acceso al trabajo de los miembros de la familia y requisitos exigidos a los familiares.

-Financiación y gestión del capital.

-Gestión de la información a los propietarios

-Procedimiento para modificar el protocolo -Etc.

En el Real Decreto 171/2007 expone que el protocolo es "aquel conjunto de pactos suscritos por los socios entre sí o con terceros con los que guardan vínculos familiares que afectan una sociedad no cotizada, en la que tengan un interés común en orden a lograr un modelo de comunicación y consenso en la toma de decisiones para regular las relaciones entre familia, propiedad y empresa que afectan a la entidad".
El protocolo familiar constituye, de esta forma, un código de conducta personal y familiar para los miembros de la familia ya que incluye pactos y pautas de comportamiento.

Según Casado (2003) es importante la formalización de un protocolo familiar, como acuerdo que delimite el marco de desarrollo y las reglas de actuación y relaciones entre la propia empresa familiar y su propiedad, sin que ello suponga interferir en la gestión de la empresa y su comunicación con terceros.

El protocolo familiar debe entenderse como el resultado de una tarea en la que participan tanto la familia como la empresa y la propiedad, que tiene componentes jurídicos, económicos y empresariales, es un documento a medida para una determinada empresa y familia Casado (2003).

\section{Conclusiones}

El protocolo es una herramienta efectiva para lograr un equilibrio entre la empresa, la propiedad y la familia. Sánchez-Crespo, Bellver y Sánchez (2005) exponen que la empresa ha de contar con una junta general y con un consejo de administración eficaz, jugando un importante papel la profesionalización de la gestión en este tipo de organizaciones (Bañegil, Barroso y Tato, 2011).

Los accionistas tienden a defender la cuenta de resultados y los gerentes pueden perseguir de forma más específica el crecimiento y la continuidad empresarial. Los conflictos pueden generarse cuando los propietarios adoptan distintas posturas ante el reparto de beneficios.

Este problema se agudiza en una empresa familiar por la interrelación de sus diversos subsistemas y la posible existencia de distintas posturas sobre el reparto, con el problema añadido de que los posibles conflictos pueden salir de la dimensión empresarial, cruzándose las cuestiones empresariales con las personales (Negreira, Negreira, 2006).

Tal y como hemos analizado en la medida en la que los distintos agentes trabajan en la empresa y tienen implicación en el control de la 
misma serán más proclives a mantener lo generado como reservas para financiar el crecimiento de la empresa y en general tienen cierta tendencia a repartir en menor cuantía dividendos.

Como consecuencia, el tener de forma específica y concreta una política de dividendos plasmada en el protocolo, los conflictos pueden minimizarse y no trascender a las diferentes dimensiones personales y familiares de este tipo de negocios.

La principal limitación del estudio es que se ha realizado un análisis no empírico de las características de la política de dividendos en la empresa familiar, recomendándose para estudios futuros estudiar a lo largo del tiempo las tendencias en las políticas de dividendos de las empresas que podemos considerar familiares.

La importancia de la empresa familiar en nuestro país junto con la vertebración de la familia y la necesidad de integración de ambos sistemas manifiestan la importancia del análisis del conflicto en la empresa familiar.

Futuros estudios podrían analizar las diferencias en el reparto de beneficios entre las empresas familiares y no familiares y el efecto de la propiedad familiar en los resultados contables y el conflicto que éstos pueden originar.

Líneas futuras de investigación podrían centrarse en la comparación las citadas variables en empresas familiares $y$ no familiares específicamente en el caso español, así como la incidencia del protocolo en la política de dividendos $\mathrm{y}$ el efecto que tiene tener una política de dividendos expresa en la reducción del conflicto en la empresa familiar.

\section{Bibliografía}

Anderson, R. Y Reeb, D.M. (2003). Founding Family Ownership and Firm Performance: Evidence from the S\&P 500, The Journal of Finance 58(3), 1301-1328.

Astrachan, J. H., Klein, S.B. \& Smyrnios, K. X. (2002). The F-PEC scale of family influence: A proposal for solving the family business definition problema. Family Business Review, 15(1), 45-58.
Astrachan, J., \& Jaskiewicz, P. (forthcoming). Emotional returns and emotional costs in privately held family businesses: Advancing traditional business valuation. Family Business Review, 21(2), 139-149.

Bhattacharya S. (1979). Imperfect Information, Dividend Policy, and "The Bird in the Hand" Fallacy, The Bell Journal of Economics, 10(1), 259270

Black, F. (1976): The dividend puzzle, The Journal of Portfolio Management, 2, 4-8.

Bañegil Palacios, T.M., Barroso Martínez, A. y Tato Jiménez, J.L. (2011). Profesionalizarse, emprender y aliarse para que la empresa familiar continúe. Revista de Empresa Familiar, 1(2), 23-37.

Basco R. (2010). Tipo de orientación familiar y prácticas de dirección y gobierno. Un estudio aplicado a las empresas familiares españolas, Revista Europea de Dirección y Economía de la Empresa, 19(2), 129-144

Benavides Velasco, C.A., Quintana García, C., Guzmán Parra, V.F. (2011). Trends in family business research. Small Business Economics. In press. DOI 10.1007/s11187-011-9362-3

Casado F. (2003). La Empresa Familiar y la necesidad de un Nuevo marco regulador para un major desarrollo functional y social en De Computis et Scripturis. Estudios en homenaje a Mario Pifarré Riera. Barcelona: Real Academia de Ciencias Económicas y Financieras.

Casillas, J. \& Acedo, F. (2007). Evolution of the intellectual structure of family business literature: a bibliometric study of FBR. Family Business Review, 20(2), 141-162.

Chrisman, J.J., Chua, J.H., Kellermanns, F.W., Matherne, C.F. III \& Debicki, B.J. (2008). Management journals as venues for publication of Family Business Research. Entrepreneurship Theory and Practice, 32(5), 927-934.

Chrisman, J. J., Chua, J. H., \& Sharma, P. (2005). Trends and directions in the development of a strategic man- agement theory of the family firm. Entrepreneurship Theory and Practice, 29(5), 555575.

Danes, S.M., Zuiker, V., Kean, R., Arbuthnot, J., \& Kaye, K. (1999). Predictors of family business tension and goal achievement. Family Business Review, 12(3), 241-252.

Demsetz, H. y Lehn, K. (1985). The Structure of Corporate Ownership: Causes and Consequences, 
Journal of Political Economy, 93, 1155-1177.

Easterbrook, F.H. (1984). Two agency-cost explanations of dividends, American Economic Review, 74(4), 650-659.

Eddleston K., Kellermanns F.W. (2007). Destructive and productive family relation-ships: a stewardship theory perspective. Journal of Business Venturing, 22(4), 545-65.

Fama, E. F. y Jensen, M. C. (1983). Agency Problems and Residual Claims, Journal of Law and Economics, 26(2), 327-349.

Fan J.P.H., Wong T. J. (2002). Corporate ownership structure and the informativeness of accounting earnigs, in Esast aAsia, Journal of Accounting and Economics, 33, 401-425.

Gallo, M.A., and Vilaseca, A. (1996). Finance in family business. Family Business Review, 9(4), 387-401.

Gordon, M. J. (1959). Dividends, Earnings and Stock Prices. Review of Economics and Statistics (The MIT Press) 41(2), 99-105.

Gugler, K. (2003) Corporate governance, dividend layout policy, and the interrelation between dividends, R\&D, and capital investment, Journal of Banking and Finance, 27, 1297-1321.

Gubler, K. Y Yortoblu, B. (2003) Corporate governance and dividend pay-out policy in Germany, American Economic Review 47(4), 731758.

Guzmán I.(2004). Factores explicativos del reparto de dividendos a cuenta en las empresas españolas, Documentos de trabajo del IVIE, Valencia: Instituto Valenciano de Investigaciones Económicas.

Handler, W. C. (1989). Methodological issues and considerations in studying family businesses. Family Business Review, 2(3), 257-276.

Harvey M, Evans R.E.(1994). Family business and multiple levels of conflict. Family Business Review, 7(4), 331-48.

Jensen, M. C. 1986). Agency Costs of Free Cash Flow, Corporate Finance, and Takeovers, American Economic Review, 76, 323-329.

Jensen, M. C. y Meckling, W. H. (1976). Theory of the Firm: Managerial Behavior, Agency Costs and Owners- hip Structure. Journal of Financial Economics, 3(4), 305-360.
Jensen, G. R., Solberg, D. P. y Zorn, T. S. (1992). Simultaneous Determination of Insider Ownership, Debt and Dividend Policies. Journal of Financial and Quantitative Analysis, 27(2), 247-263.

Jian-ping, D. y Young, Z. (2005). A study on the relationship between the family control and vividend decision of the listed company, www.ccfr.org.cn. .

Kellermanns FW, Eddleston K. (2004). Feuding families: when conflict does a family firm good. Entrep Theory Pract, 28(3), 209-248.

Kellermanns, F.W., Kimberly A., Eddleston (2007). A family perspective on when conflict benefits family firm performance, Journal of Business Research 60, 1048-1057.

La Porta, R., López de Silanes, F., y Shleifer, A. (1999). Corporate ownership around the World, The Journal of Finance, 54, 471-517

Lang y Litzenberger (1989). Dividend Announcements. Cash Flow Signaling Vs. Free Cash Flow Hypothesis. Journal of Financial Economics, 24, 181-191.

Lee M-S, Rogoff EG (1996). Comparison of small businesses with family participation versus small businesses without family participation: an investigation of differences in goals, attitudes, and family/business conflict. Family Business Review, 9(4), 423-37.

Levinson H. (1971). Conflicts that plague family businesses. Harvard Business Review, 49, 90-8.

Levinson RE.(1987). Problems in managing a family-owned business. In: Aronoff CE, Ward JL, editors. Family business sourcebook. Detroit: Omnigraphics, Inc.

Litz, R.A. (1995). The family business: Toward a definitional clarity. Academy of Management Journal Best Papers Proceedings, 100-104.

Lozano, M. B., Miguel, A. y Pindado J. (1998). Decisiones de inversión en la empresa: un nuevo enfoque para su análisis. Economía Industrial, 324, VI, 123-132.

Morck, R., Shleifer, A., Vishny, W. (1998). Management ownership and market valuation: an empirical análisis. Journal of Financial Economics, 20, 292-315.

Miller, D., Le Breton-Miller, I., Lester, R.H., Cannella Jr., A.A., 2007. Are family firmsvreally superior performers? Journal of Corporate Finance 13, 829-858. 
Miller, M. H. and Modigliani, F. (1961). Dividend Policy, Growth and the Valuation of Shares. Journal of Business, 34(4), 11-33.

Negreira del Río, J. y Negreira del Río, F. (2006). El reparto de dividendos en la Empresa Familiar. Harvard Deusto Finanzas y Contabilidad, 72, 64-70.

Neubauer, F. \& Lank, A. (1998). The family business: Its governance for sustainability. London: MacMillan Press.

Noronha, G. M., Shome, D. K. y G. E. Morgan (1996). The Monitoring Rationale for Dividends and the Interactions of Capital Structure and Dividend Decisions, Journal of Banking and Finance, 20, 439-454.

Poterba, J.M. y Summers L. H. (1984). New Evidence that Taxes Affect the Valuation of Dividends. The Journal of Finance, 39, 1397-1415.

Rosenblatt, P. C. (1991). The interplay of family sys- tems and business systems in family farms during economic recession. Family Business Review, 4(1), 45- 57.

Rozeff, Michael S. (1982). Growth, beta and agency costs as determinants of dividend payout ratios. The Journal of Financial Research 5, 249259.

Sabater R. (2010). El reparto de beneficios en la empresa familiar, Laverdad.es, 13/10/10 http://www.laverdad.es/murcia/v/20101013/opinion/ reparto-beneficios-empresa-familiar-20101013.html

Sadler, P. (1990). Strategic sense and family sensibility. Director, 43(11), 27-29.

Sánchez-Crespo, A. J., Bellver, A. y Sánchez, A. M. (2005). La empresa familiar: manual para empresarios, Barcelona: Ediciones Deusto.

Sánchez-Crespo, A.J. (2003). El cambio generacional en la Empresa Familiar, Boletín del ilustre colegio de abogados de Madrid, 27.

Santana, D.J. y Cabrera M.K. (2001). Comportamiento y resultados de las empresas cotizadas familiares versus no familiares, IX Congreso Nacional de ACEDE.

Schweiger DM, Sandberg WR, Rechner PL.(1989). Experiential effects of dialectical inquiry, devil's advocacy, and consensus approaches to strategic decision making. Acadedy Management Journal, 32, 757-72.

Shanker, M. C. \& Astrachan, J. H. (1996). Myths and Realities: family Businesses' Contribution to the US Economy-A Framework for Assessing Family Business Statistics. Family Business Review, 9(2), 107-119.

Sharma P, Chrisman JJ, Chua JH. (1997). Strategic management of the family business: past research and future challenges. Family Business Review, 10(1). 1-36.

Stavrou, E.T. (1999). Succession in family businesses: exploring the effects of demo- graphic factors on offspring intentions to join and take over the business. Journal of Small Business Management 37(3), 43-61.

Tagiuri, R., \& Davis, J. A. (1992). On the goals of successful family companies. Family Business Review, 5(1), 43-62.

Tagiuri, R., \& Davis, J. A. (1996). Bivalent attributes of the family firm. Family Business Review, 9(2), 199- 208.

Walsh JP, Fahey L. (1986). The role of negotiated belief structures in strategy making. Journal Management, 12(3), 325-380.

Wortman, M. S., Jr. (1994). Theoretical foundations for family-owned business: A conceptual and research-based paradigm. Family Business Review, 7(1), 3-27. 